\title{
Mir-29b Regulates Oxidative Stress by Targeting SIRT1 in Ovarian Cancer Cells
}

\author{
Meng Hou Xiaohang Zuo $^{\mathrm{b}}$ Chen Lia Yan Zhang ${ }^{\mathrm{c}} \quad$ Yue Teng ${ }^{\mathrm{a}}$ \\ aDepartment of Obstetrics and Gynecology, The First Affiliated Hospital of Xi'an Jiaotong University, \\ Xi'an, bNo.323 Hospital of Chinese People's Liberation Army, Xi'an, 'Center for Translational Medicine, \\ The First Affiliated Hospital of Xi'an Jiaotong University, Xi'an, China
}

\section{Key Words}

Micro-RNAs • SIRT1 (silent mating type information regulation 2 homolog 1 ) $\cdot$ ROS (reactive oxygen species) $\bullet$ Ovarian Cancer

\begin{abstract}
Background: Metabolic abnormalities are frequently observed in multiple malignancies including epithelial ovarian cancer (EOC), among which imbalance between generation and elimination of reactive oxygen species (ROS) plays a critical role in EOC onset and progression. Here we investigated the role of miR-29b, a well-established tumor-suppressor miRNA in metabolic regulation of EOC cells. Methods: cell viability and apoptosis in miR-29b inhibited and over-expressed EOC cells were evaluated by CCK8 and Annexin V-FITC/PI assays. Change in miR-29b was detected in EOC cells incubated in $\mathrm{H}_{2} \mathrm{O}_{2}$ culture by q-PCR. Relative ROS levels were also detected in different EOC cultures, including modified miR-29b and SIRT1 levels as well as $\mathrm{H}_{2} \mathrm{O}_{2}$ incubation. A luciferase reporter assay was employed to detect the direct binding of miR-29b to SIRT1 3' UTR. Changes in cell viability and ROS levels were assessed in SIRT1knocked down EOC cells. Results: miR-29b expression correlates with decreased EOC cell viability and increased apoptosis. $\mathrm{H}_{2} \mathrm{O}_{2}$ downregulated miR-29b in a time and dose-dependent manner. miR-29b expression negatively correlated with ROS levels, whereas SIRT1 significantly stimulated ROS formation. Luciferase reporter assays confirmed miR-29b downregulation of SIRT1by directly targeting its mRNA 3'-UTR. SIRT1 silencing rescues cell viability of $\mathrm{H}_{2} \mathrm{O}_{2}$ treated cells. Also, SIRT1 inhibition blocked cell apoptosis induced by $\mathrm{H}_{2} \mathrm{O}_{2}$ as well as reduced intracellular ROS levels. Conclusion: Together, our findings indicated that the miR-29b/SIRT1 axis has a protective effect against $\mathrm{H}_{2} \mathrm{O}_{2}$-induced damage of cell viability and oxidative stress and may provide novel options for miR-29b-based therapeutic approaches for EOC treatment.

(C) 2017 The Author(s)

Published by S. Karger AG, Basel

\section{Introduction}

Epithelial ovarian cancer (EOC) is the most lethal gynecological malignancy, and is the fifth most common cause of cancer death in women [1] . Most EOC patients are diagnosed M. Hou, X. Zuo and C. Li contributed equally to this work.

Yue Teng and Yan Zhang

Department of Obstetrics and Gynecology and Center for Translational Medicine,

The First Affiliated Hospital of Xi'an Jiaotong University, Yanta West Road 277, Xi'an, (China); Tel.+8602985323840, E-Mail navimoon@126.com and E-Mail zyyan1114@xjtu.edu.cn
\end{abstract}

KARGER 
at late stages, with a 5-year survival rate of less than 30\%[2]. Current standard treatment for EOC includes a combination of cytoreductive surgery and platinum-based chemotherapy, which acts efficiently as initial treatment. However, as treatment continues, most EOC patients recur and turn to be resistant to existing treatments [3]. Therefore, alternative approaches to the diagnosis and treatment of EOC patients are urgently needed. Exploration of novel biomarkers for early diagnosis, prognosis prediction, and effective therapies will definitely contribute to the current state of clinical care of EOC.

Reactive oxygen species (ROS) are a series of highly reactive molecules principally derived from the oxygen that is consumed in various metabolic activities occurring mainly in the mitochondria, peroxisomes, and the endoplasmic reticulum [4]. Regulation of intracellular ROS is critical for cellular homeostasis, since different ROS levels could lead to different biological responses.ROS at low and moderate levels acts as signaling molecules that sustain cellular proliferation, differentiation, and cell cycle progression as well as activate stress responsive survival pathways [5]. However, excessive ROS levels triggers severe cell damage and apoptosis pathways [6]. Accumulating evidence shows that excess generation of ROS elicits oxidative stress in cells and tissues and leads to various diseases, including various cancers [7-9]. Accumulated oxidative stress leads to a rise in ROS levels, which in hence activate oncogene signaling pathways and may further exploit underlying mutagenesis and genomic variability in cancer cells to stimulate cancer progression [10]. Increased ROS production is also a hallmark of EOC and ROS is suggested to be prosurvival and antiapoptotic in ovarian cancer cells [11].

SIRT1 belongs to the sirtuins family of NAD+-dependent histone/protein deacetylases, which has been frequently reported to regulate diverse cellular processes including antioxidative stress, cell differentiation, cell survival, cell cycling, and metabolism by deacetylating downstream targets [12]. SIRT1 has been found to be expressed in various tissues, including ovaries [13]. In our previous studies, we focused on exploring the versatile roles of miR-29b in EOC onset and progression, and found that miR-29b plays vitals roles in regulating energy metabolism, mesenchymal transition, as well as epigenetic regulation of ovarian cancer cells [14-16]. Noticeably, using bioinformatics, we previously identified SIRT1 as potential downstream target gene of miR-29b, indicating that miR-29b is probably involved in cancerous ROS manipulation by targeting SIRT1 signaling pathway. However, so far there is no research available on whether miR-29b plays a role in the oxidative stress during ovarian cancer. We believe that elaborating both the specific roles of miR-29b in the oxidative stress of ovarian cancer and the latent molecular mechanisms will further our theoretical understanding of human ovarian cancer as well as provide future clinical approaches to treating EOC.

\section{Materials and Methods}

Cell lines

The human ovarian cancer cell lines SKOV3 and A2780(both obtained from ATCC, Manassas, VA,USA) were maintained in high-glucose DMEM (Gibco,Invitrogen, Carlsbad, CA, USA) supplemented with 10\%(v/v) fetal bovine serum at $37^{\circ} \mathrm{C}$ in a humidified $5 \% \mathrm{CO}_{2}$ atmosphere.

\section{Bioinformatics analysis}

Potential targets and binding sites of miR-29b werepredicted using several online programs, includingmiRanda(http://www.microrna.org/microrna/home.do),miRBase (http://www.mirbase. org/index.shtml), miRWalk(http://www.umm.uni-heidelberg.de/apps/zmf/mirwalk/index.html), and TargetScan (http://www.targetscan.org/); SIRT1 was predicted to be a downstream targetgene of miR-29b by all of these softwares, and this wasfurther validated by experiments.

Cell transfection

The control mimics, miR-29b mimics, control inhibitors, and miR-29b inhibitors were all purchased from RiboBio (Guangzhou, China). SIRT1targeted siRNAs, SIRT1 adenoviruses (SIRT1-Ad), and their respec- 


\section{Cellular Physiology Cell Physiol Biochem 2017;43:1767-1776

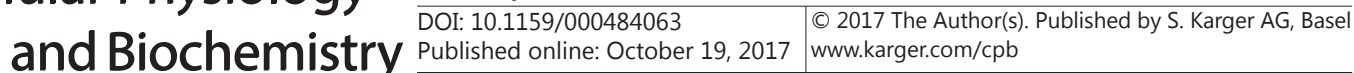 \\ Hou et al.: Interaction of Mir-29b and SIRT1 in EOC}

tive negative controls were purchased from GenePharma(Shanghai, China). Transfection of ovarian cancer cells were conducted as described previously [14]. $48 \mathrm{~h}$ after transfection, the cells were collected for further experiments.

Quantitative real-time PCR

qPCR was performed as depicted before [10]. In brief, total RNA was collected using TRIzol reagent(Invitrogen, Carlsbad, CA, USA). First-strand cDNA was synthesized usingPrimeScript RT reagent kit(Perfect Real Time; Takara, Dalian, China). qPCR was then performed using SYBR PremixEx Taq ${ }^{\mathrm{TM}}$ II (Takara) on a CFX96 real-time PCR System(Bio-Rad, Hercules, CA, USA). $\beta$-actin was used as an internal control for results normalization.miR-29b levels were detected using a TaqMan microRNA kit (Applied Biosystems) and normalized to small nuclear RNA (Rnu6),which was taken as a control. The data were expressed as Log 2-fold changes relative to miRs/U6 snRNA levels. The primers for miR-29b and U6 reverse transcription and amplification were designed by and purchased from RiboBio Co., Ltd. (Guangzhou, China). All primers used are listed in Table 1.

\section{Western blot analysis}

Cell lysates were collected using mammalian protein extraction reagent (Pierce, Rockford, IL, USA) with protease inhibitors (Roche, Indianapolis, IN,USA). The protein concentration of each sample was determined using a BCA-200 protein assay kit (Pierce, Rockford, IL, USA). The proteins were resolved on 10\% SDS-polyacrylamide gels and transferred onto nitrocellulose membranes. The membranes were blocked in blocking buffer (5\% non-fat milk in TBST). Mouse anti-humanSIRT1 (ab110304, Abcam, Cambridge, UK), and mouse anti-human $\beta$-actin (\#3700S, CST, MA, USA) were incubated with the membranes overnight at $4^{\circ}$ Cat dilutions of 1:800, and 1:2000,respectively. After washed with TBST, the blots were visualized with anti-rabbit or anti-mouse IgG conjugated with peroxidase (HRP) and ECL reagents (Pierce, Rockford, IL, USA).

\section{Cell viability assay}

The viability of EOC cells was determined using the cell counting kit-8 (CCK-8) assay (Beyotime Institute of Biotechnology, Beijing, China), according to the manufacturer's protocols. Briefly, cells were seeded into 96-wellplates (3000 cells/well). After being incubated in $10 \mu \mathrm{l} \mathrm{CCK-8for} 2$ hours, the absorbance of each well was measured at $450 \mathrm{~nm}$ (Tecan Infinite M200 microplatereader; LabX, Austria). All of the experiments were performed at leastin triplicate.

\section{Measurement of apoptosis}

To detect cell apoptosis, EOC cells were stained with AnnexinV-fluoresce in isothiocyanate (FITC) and counterstained with propidium iodide (PI) using the Annexin V-FITC/PIapoptosis detection kit (BD PharMingen, San Diego, CA,USA), according to the manufacturer's protocols. Briefly, the cells were treated as indicated, trypsinized, and harvested by centrifugation. Then, the cells were incubated with annexin $\mathrm{V}$ and PI for $15 \mathrm{~min}$ at room temperature in the dark. Analyses were performed using FACScan cytometer (BD Biosciences, Woburn, MA,USA) and FlowJo software (BD Biosciences).

\section{Intracellular Reactive Oxygen Species (ROS) Assay}

EOC cells were grown to confluence in a 24-well plate and then transfected with miRNAs, siRNAs, and adenoviruses, and cultured for $48 \mathrm{~h} . \mathrm{H}_{2} \mathrm{O}_{2}(400 \mu \mathrm{M})$ was added for the last $2 \mathrm{~h}$. The cells were then labeled with 2',7'-dichlorofluorescin-diacetate(DCFH-DA, Sigma) at $37 \circ \mathrm{C}$ for $30 \mathrm{~min}$ and readings were performed using FACScan cytometer (BD Biosciences, Woburn, MA,USA). 


\section{Cellular Physiology Cell Physiol Biochem 2017;43:1767-1776 \begin{tabular}{l|l|l} 
and Biochemistry Published online: October 19, 2017 & $\begin{array}{l}\text { (c) } 2017 \text { The Author(s). Published by S. Karger AG, Basel } \\
\text { www.karger.com/cpb }\end{array}$
\end{tabular}}

Luciferase reporter assay

Luciferase reporter assay was performed as depicted before [15]. Briefly, The SIRT1 3'UTR containing the predicted miR-29b target sequence was amplified from EOC cell genomic DNA and cloned into the pGL3 firefly luciferase control vector (Promega, Madison, WI) at the XhoI restriction site directly downstream of the reporter gene. To generateSIRT1 3'UTR with a mutant target sequence, transversion mutations of 7 nucleotides were made at themiR-29b seed region complementary sites as shown in Fig. 3C. Inhibition of the luciferase reporter gene levels by miR-29b was assessed in SKOV3and A2780 cells as indicated before [15]. The results were manifested as percentage change over the respective control.

\section{Statistical analysis}

All of the experiments were performed at least in triplicate and each experiment was independently performed at least 3 times. Data were presented as mean \pm standard deviation (SD) and analyzed using GraphPad Prism 5 software. Statistical significance was assessed using two-tailed unpaired Student's t-test. When a P value was less than 0.05 , the differences were considered as statistically significant.

\section{Results}

miR-29b expression correlates with decreased EOC cell viability and increased apoptosis.

As illustrated before, miR-29b has been found to be a versatile player in EOC onset and progression, correlated with cell differentiation, migration, energy metabolism, as well as epigenetic regulation [14-16]. Here, CCK-8 assay was taken to evaluate the role of miR-29b in EOC cell survival. As shown in Fig. 1, over-expression of miR-29b reduced cell survival, while inhibition of miR-29b expression enhanced cell survival in both EOC cell lines (Fig. 1A). According to flow cytometric analysis (Fig.1B), miR-29b mimic-treated EOC cells were significantly more apoptotic than their NC mimics counterparts. However, miR-29b inhibition via target inhibitor transfection led to a decrease in EOC cell apoptosis. These results support our hypothesis that overexpression of miR-29b correlates with decreased EOC cell viability and increased apoptosis.

\section{Effects of $\mathrm{H}_{2} \mathrm{O}_{2}$ on miR-29b expression.}

Among the various ROS ingredients, $\mathrm{H}_{2} \mathrm{O}_{2}$ is the most stable and diffusible form and thus frequently taken to represent cellular ROS level. Balance of cellular $\mathrm{H}_{2} \mathrm{O}_{2}$ is decisive for cell fate: low and intermediate levels of $\mathrm{H}_{2} \mathrm{O}_{2}$ stimulate loss of cell homeostasis and increased
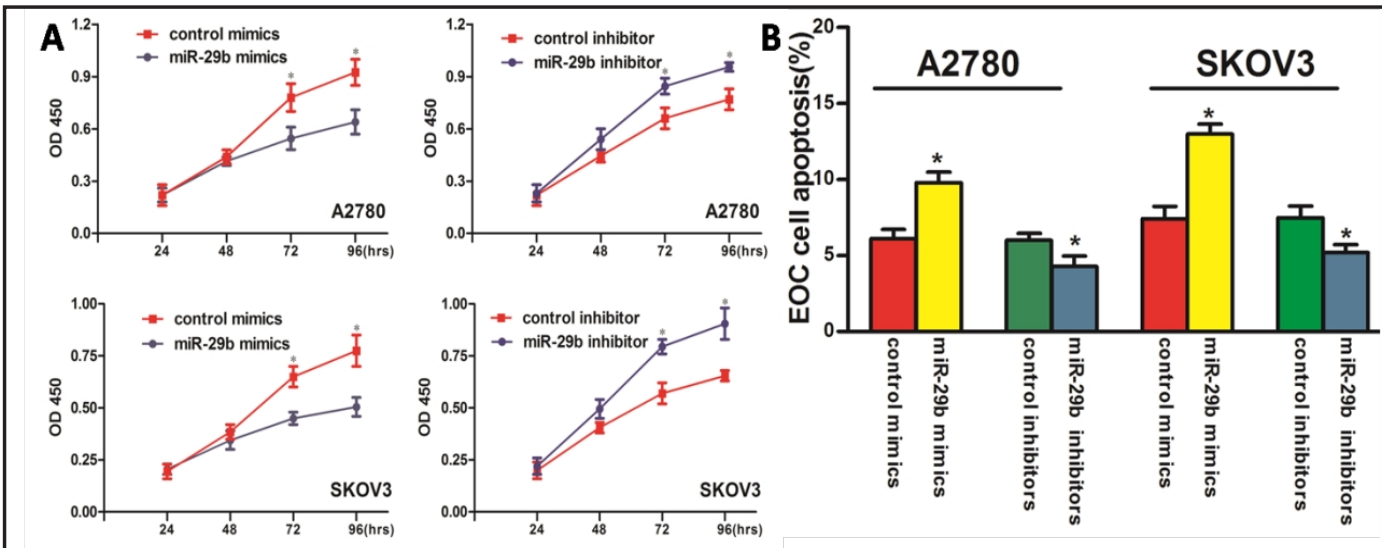

Fig. 1. miR-29b expression correlates with decreased EOC cell viability and increased apoptosis. A) A2780 and SKOV3 cells were transfected as indicated, and cell viability at indicated time points was analyzed by CCK8 assay. The optical density at the wave length of $450 \mathrm{~nm}$ (OD 450) was positively associated with the count of viable cells. (B) Flow cytometric analysis of apoptotic cells in cultures of miR-29b mimics, NC mimics, miR-29b inhibitors, or NC inhibitors treated (A2780 and SKOV3 cells were transfected as indicated). $\mathrm{NC}$ : negative control, PI propidium iodide. Data are presented as means $\pm \mathrm{SEM}, \mathrm{n}=3 .{ }^{*} \mathrm{p}<0.05$ versus control. 
Fig. 2. Effects of $\mathrm{H} 2 \mathrm{O} 2$ at different concentrations and time points on miR-29b expression. A) The expression of miR-29b was detected by q-RT PCR in A2780 and SKOV3 cells treated with the indicated concentration of $\mathrm{H}_{2} \mathrm{O}_{2}$ $(0,5,10$, and $20 \mu \mathrm{M}$, respectively) for $6 \mathrm{~h}$. B) The expression of miR$29 \mathrm{~b}$ was detected by q-RT PCR in A2780 and SKOV3 cells treated with $\mathrm{H}_{2} \mathrm{O}_{2}$ at a concentration of $10 \mu \mathrm{M}$ at the indicated time points. C) Intracellular levels of ROS triggered by pre-incubation exposure of A2780 and SKOV3 cells to H2O2, miR-29b mimics, miR-29b inhibitors, si-SIRT1, or SIRT1-Ad were assessed by DCF assays. Data are presented as means \pm SEM, $n=3 .{ }^{*} \mathrm{p}<0.05$ versus control.

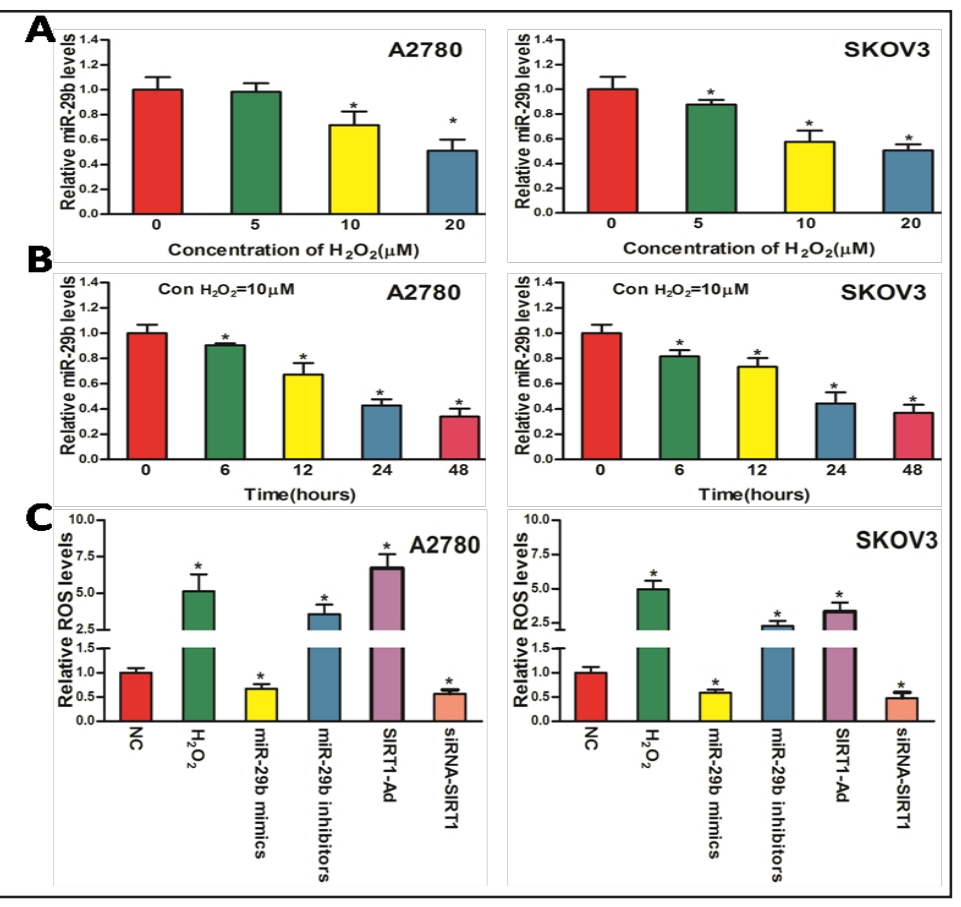

adaptation to stress which leads to neoplastic transformation while high levels of $\mathrm{H}_{2} \mathrm{O}_{2}$ induce cell death [4]. In order to test the correlation between miR-29b and ROS in EOC cells, here we evaluated the expression of miR-29b in both EOC cell lines treated with $\mathrm{H}_{2} \mathrm{O}_{2}$ for different periods of time. As indicated in Fig. $2 \mathrm{~A}, \mathrm{H}_{2} \mathrm{O}_{2}$ downregulated the expression of miR-29b in a time-dependent manner. To find out whether $\mathrm{H}_{2} \mathrm{O}_{2}$ mediated miR-29b expression in a dosedependent manner, we then treated EOC cells with $\mathrm{H}_{2} \mathrm{O}_{2}$ at different concentrations. $\mathrm{H}_{2} \mathrm{O}_{2}$ decreased the expression of miR-29b when incubated in $\mathrm{H}_{2} \mathrm{O}_{2}$ at a concentration of $10 \mu \mathrm{M}$ compared to their control counterparts (Fig. 2B). These results suggest that $\mathrm{H}_{2} \mathrm{O}_{2}$ reduced miR$29 \mathrm{~b}$ expression in EOC cells in a time- and dose-dependent manner. Further, we determined the effect of $\mathrm{H}_{2} \mathrm{O}_{2}$, miR-29b as well as SIRT1, a potent downstream target of miR-29b on ROS formation in EOC cells. As indicated in Fig. 2C, $\mathrm{H}_{2} \mathrm{O}_{2}$ incubation dramatically enhanced cellular ROS generation, miR-29b expression negatively correlated with ROS levels, whereas SIRT1 significantly stimulated ROS formation (Fig. 2C).

miR-29b directly targets negatively SIRT1.

Intriguingly, modifications in miR-29b and SIRT1 led to opposite changes in ROS levels (Fig. 2C), which encouraged us to explore the underlying connection between miR-29b and SIRT1. We employed micro-RNA mimics and inhibitors to specifically overexpress and knock down the endogenous expression of miR-29b in A2780 as well as SKOV3 cells. As shown in Fig. 3A and 3B, SIRT1 expression was significantly decreased by transfection with miR-29b mimics and was greatly increased by transfection with miR-29b inhibitors at both the mRNA and protein level. Therefore, miR-29b negatively regulates SIRT1 expression in both selected EOC cell lines. We then employed bioinformatics software (including miRanda,Targetscan, miRBase, and miRWalk) for a prediction of miR-29b downstream targets. By analyzing the 3'-UTR sequence of SIRT1 as well as the mature chain sequence of miR-29b, we found that the seed region in the miR-29b mature chain is fully complementary with and thus potentially binds to the SIRT1 3'UTR sequence (Fig. 3C). This observation suggested that miR-29b might negatively regulate SIRT1 level by directly binding to its 3'UTR sequences. Finally, the direct binding between miR-29b and SIRT1 3'UTR was confirmed by the 3'UTR luciferase reporter assay. In brief, ovarian cancer cells were transfected with control or 


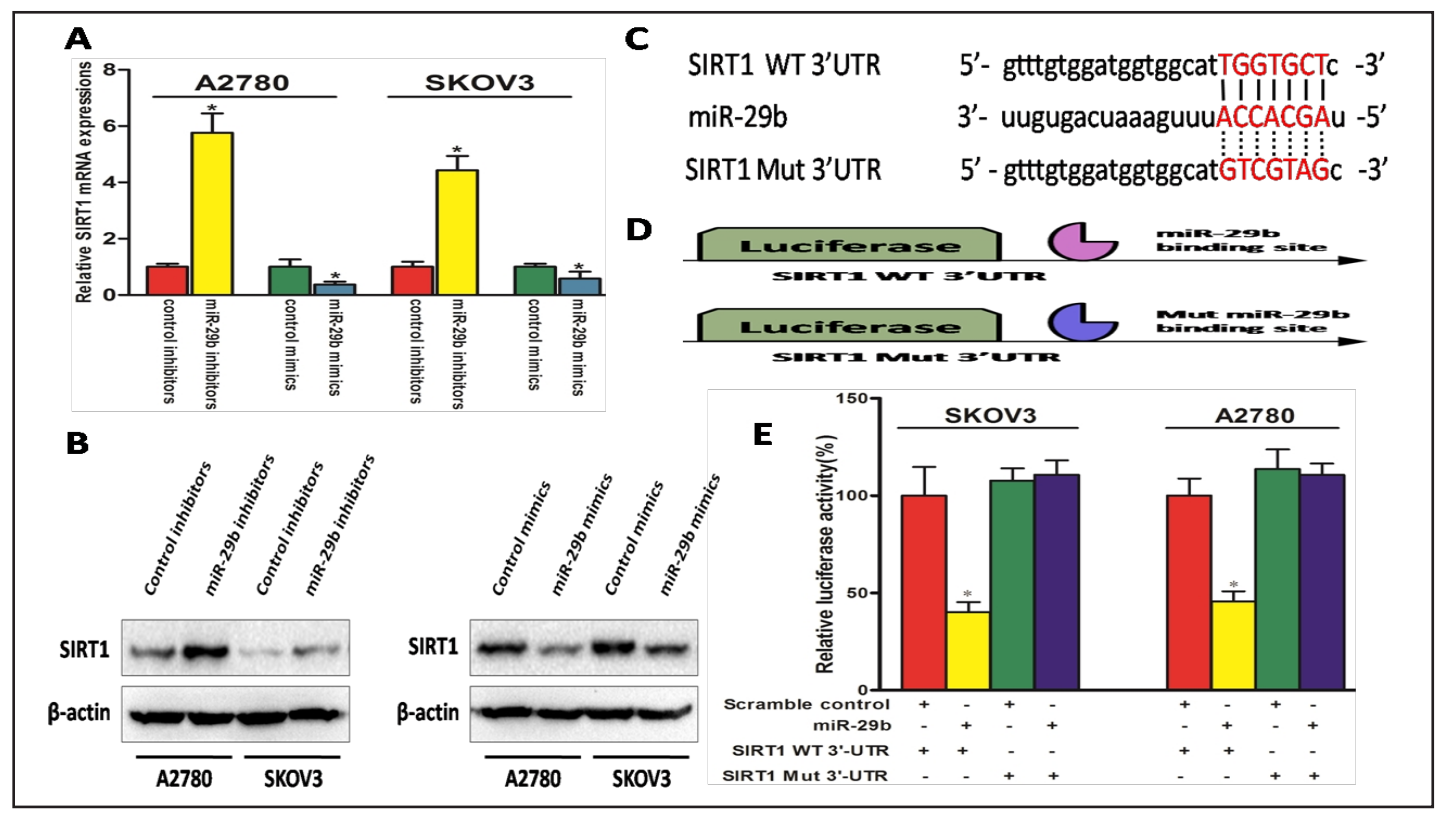

Fig. 3. miR-29b directly targets and thus negatively regulates SIRT1. A) and B) qPCR(A) and Western(B) results show that SIRT1 expression was significantly decreased by miR-29b over-expression and greatly increased by miR-29b inhibition at both the mRNA and protein levels in both A2780 and SKOV3 cells. C) and D) Schematic representation of different SIRT1 3'UTR luciferase reporters used in the transfection experiments are depicted. E) A2780/SKOV3 cells were co-transfected with either miR-29b or control mimics and $200 \mathrm{ng}$ pGL3 reporter construct containing wild type or miR-29b site mutated SIRT1 3'UTR. The relative firefly luciferase activities normalized with renilla luciferase were measured 48 hours post-transfection and results are plotted as percentage change over respective controls. Data are presented as means \pm SEM, $n=3$. $* \mathrm{p}<0.05$ versus control.

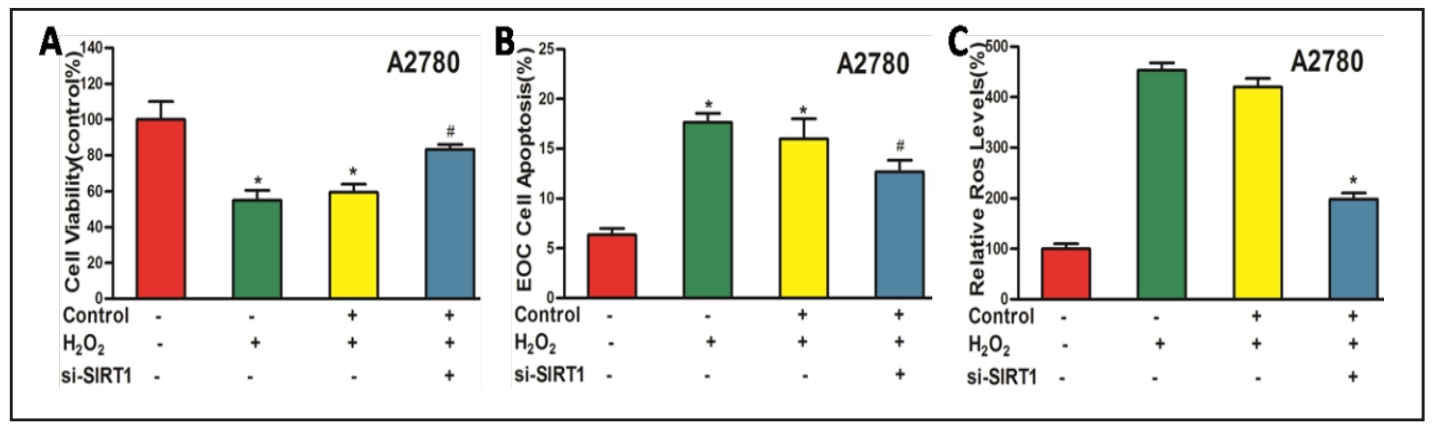

Fig. 4. siRNA-mediated downregulation of SIRT1 enhanced the viability and attenuated ROS production in ovarian cancer cells treated with $\mathrm{H}_{2} \mathrm{O}_{2}$.A) The CCK-8 assay was performed to evaluate the cell viability of A2780 cells treated as indicated. B) An apoptosis assay was used to assess the apoptosis levels of A2780 cells treated as indicated. C) Intracellular formation of ROS was measured in A2780 cells treated as indicated. Data are presented as means $\pm \mathrm{SEM}, \mathrm{n}=3$. ${ }^{*} \mathrm{p}<0.05$ versus control; and ${ }^{*} p<0.05$ versus $\mathrm{H}_{2} \mathrm{O}_{2}$ group.

miR-29b mimics together with a luciferase construct containing either the wild-type SIRT1 3'UTR or a mutant SIRT13'UTR (Fig. 3D). Only transfection of the wild-type SIRT1 3'UTR significantly decreased luciferase expression, and this suppressive effect of miR-29b was later abolished by mutating the miR-29b site in SIRT1 3'UTR (Fig. 3E). Collectively, miR-29b was observed to directly bind to its complementary sequence motifs in the SIRT1 3'UTR, thus negatively regulating its level. 


\section{Cellular Physiology Cell Physiol Biochem 2017;43:1767-1776

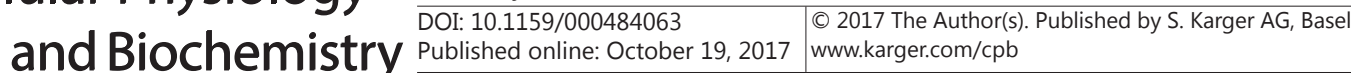 \\ Hou et al.: Interaction of Mir-29b and SIRT1 in EOC}

SiRNA-mediated downregulation of SIRT1 enhanced the viability and attenuated ROS production in ovarian cancer cells.

In the previous experiment, we observed $\mathrm{H}_{2} \mathrm{O}_{2}$ downregulated the expression ofmiR$29 \mathrm{~b}$ and miR-29b/SIRT1 direct targeting, next we explore whether SIRT1 plays a role in miR$29 \mathrm{~b}$-induced EOC cells viability and oxidative stress. We downregulated SIRT1 expression in A2780 cells treated with $\mathrm{H}_{2} \mathrm{O}_{2}$ using siRNA transfection. As demonstrated in Fig. 4A, cell viability was rescued under the $\mathrm{H}_{2} \mathrm{O}_{2}$ treatment in siRNA-mediatedSIRT1knocked-down cells. Besides, SIRT1 inhibition also blocked the cell apoptosis induced by $\mathrm{H}_{2} \mathrm{O}_{2}$ (Fig. 4B). Further, SIRT1 knockdown reduced intracellular ROS levels (Fig. 4C). Together, our findings indicated that the miR-29b/SIRT1 axis has a protective effect agains $\mathrm{tH}_{2} \mathrm{O}_{2}$-induced damage of cell viability and oxidative stress.

\section{Discussion}

Our results show that overexpression of miR-29b is strongly associated with lower viability and increased apoptosis in EOC cells. miR-29b is also found to be decreased in a ROS-dependent manner. This study strongly suggests that miR-29b is a promising candidate for a more optimized target for EOC therapy.

In recent decades, various roles of miRNAs have been explored in diverse physiological and pathological activities, including tumorigenesis [17-21]. For instance, miR-34a has been observed to mediate multiple cancerous pathways by targeting crucial genes in tumorigenesis including P53, PDGFR, and c-MET, etc [22]. miR-29b, a well-established tumor-suppressor miRNA, has been found to be dysregulated in various human cancers, including ovarian cancer [14-16, 23]. In the current study, we showed miR-29b's role in modulating cell viability and apoptosis. A negative correlation was observed between miR$29 \mathrm{~b}$ and SIRT1, a crucial regulator in cellular oxidative stress. We further confirmed that miR-29b regulates SIRT1 by directly targeting its 3'UTR sequences, which adds a novel role for miR-29b as a ROS regulator in EOC development. Of note, our data are in agreement with previous reports demonstrating that miR-29s is capable of regulating SIRT1 in agerelated hearing loss and self-renewal of mouse embryonic stem cells, adding a novel role of the tumor suppressor miRNA miR-29b in cancerous oxidative stress [24, 25]. Intriguingly, recent studies have observed a panel of differentially expressed miRNAs in urines of ovarian cancer patients as compared to healthy control [26]. Superior to blood to some extent, urine is the ideal bio-fluid for the biomarker detection for it allows non-invasive collection. Thus, further study may focus on detecting the specific expression patterns of miR-29b in urines of EOC patients and control, aiming to explore the possibility of miR-29b as a non-invasive biomarker for EOC screening.

ROS are byproducts of biological reactions of energy generation, which are mainly produced in the mitochondria throughout the process of oxidative metabolism $[27,28]$. The balance between ROS generation and elimination is crucial for cell survival, while imbalance of ROS level triggers oxidative damage which in hence exerts discrepant impacts on cancer cell survival and causes final cell death by various mechanisms. Since ROS are generated during ovulation, oxidative stress has been proposed as a cause of ovarian cancer [29]. Actually, the association between ovarian cancer and oxidative stress has been repeatedly identified in the past decades [30-32]. Compared with other ROS, $\mathrm{H}_{2} \mathrm{O}_{2}$ is a relatively longlived molecule commonly used to represent cellular ROS levels. As demonstrated in our study, incubation of $\mathrm{H}_{2} \mathrm{O}_{2}$ dramatically enhanced cellular ROS level, indicating that the dynamic oxidant/antioxidant balance is perturbed following the addition of exogenous $\mathrm{H}_{2} \mathrm{O}_{2}$ to cultured cells. Also, treatment of EOC cells with $\mathrm{H}_{2} \mathrm{O}_{2}$ caused a dose and time-dependent decrease in miR-29b levels of EOC cells, suggesting miR-29b close involvement in EOC oxidative metabolism. Accumulating studies have revealed that ROS can alter the expression of certain miRNAs $[33,34]$. The mechanism underlying ROS-regulated microRNA expression is complicated and poorly understood. The major proposed mechanisms so far include that 
ROS regulates microRNA biogenesis enzymes, through transcription factors, and through epigenetic modification [35]. Further exploration is needed to reveal underlying mechanism of ROS regulation of miR-29b in EOC cells.

SIRT1, identified as a direct downstream target of miR-29b, belongs to the Sirtuins family of $\mathrm{NAD}^{+}$-dependent histone deacetylases, which controls cellular activities and maintains metabolic homeostasis by reducing apoptosis, attenuating inflammation, and modulating oxidative stress [36]. SIRT1 activation led to beneficial effects in metabolic disorders involving diabetes, cardiovascular disease, as well as tumorigenesis [37, 38]. Hence, regulating SIRT1 should be beneficial in treating cancerous diseases. Indeed, regulation of SIRT1 by miRNAs has been suggested [39]. For instance, miR-9 directly targets and lowers SIRT1 expression, leading to inhibited cell proliferation in AE-positive AML cell lines [40]. miR-141 targets Sirt1 and inhibits autophagy to reduce HBV replication, which could be taken as a target to lower the possibility of developing liver cirrhosis and hepatocellular carcinoma [41]. In this study, we observed a decrease in miR-29b levels in $\mathrm{H}_{2} \mathrm{O}_{2}$-treated EOC cells. miR-29b and SIRT1 exerted opposite effects on cell viability. Luciferase assays later determined the direct targeting of SIRT1 by miR-29b. Decreased SIRT1 expression attenuated ROS production in $\mathrm{H}_{2} \mathrm{O}_{2}$-treated EOC cells. SIRT1 has been found to regulate various well-established tumorigenic pathways, like Wnt- $\beta$ catenin and PI3K/Akt [42-44]. Intriguingly, in our previous study, we have revealed miR-29b direct targeting and negative regulation of AKT2/3 and their roles in aberrant EOC metabolism [15]. Thus, interaction between miR-29b, AKT2/3, and SIRT1 and the underlying molecular mechanism DNMT3A/3B-Id-1 interaction, as well as their shares in EOC development call for further exploration in future study.

\section{Conclusion}

The data obtained in the present study indicated that with a complementary structure, miR-29b combines and thus negatively regulates SIRT1. miR-29b expression correlates with EOC cell viability and apoptosis, which may be regulated by ROS production. The findings together raised the possibility that miR-29b/SIRT1axis may be potent options for future clinical treatments of ovarian cancer.

\section{Disclosure Statement}

The authors declare that no potential conflicts of interest exist.

\section{Acknowledgements}

This study was supported by grants from the National Natural Science Foundation of China (Nos. 81402313 and 81670806) and the Fundamental Research Funds for the Central Universities (No. xjj2016111).

\section{References}

1 Siegel RL, Miller KD, Jemal A: Cancer statistics, 2016. CA Cancer J Clin 2016;66:7-30.

2 Shapira I, Oswald M, Lovecchio J, Khalili H, Menzin A, Whyte J, Dos SL, Liang S, Bhuiya T, Keogh M: Circulating biomarkers for detection of ovarian cancer and predicting cancer outcomes. Br J Cancer 2014;110:976-983.

3 Samaille F, Chantant M, Houtte DV, Cordier JJ, Gargiulo L: First-line treatment of advanced ovarian cancer: current research and perspectives. Expert Rev Anticancer Ther 2010;10:47. 


\section{Cellular Physiology Cell Physiol Biochem 2017;43:1767-1776 \begin{tabular}{l|l|l} 
and BOI: 10.1159/000484063 & $\begin{array}{l}\text { C } 2017 \text { The Author(s). Published by S. Karger AG, Basel } \\
\text { www.karger.com/cpb }\end{array}$ \\
\hline
\end{tabular}}

Hou et al.: Interaction of Mir-29b and SIRT1 in EOC

4 Marengo B, Nitti M, Furfaro AL, Colla R, Ciucis CD, Marinari UM, Pronzato MA, Traverso N, Domenicotti C: Redox Homeostasis and Cellular Antioxidant Systems: Crucial Players in Cancer Growth and Therapy. Oxid Med Cell Longev 2016;2016:6235641.

-5 Janssen-Heininger YMW, Mossman BT, Heintz NH, Forman HJ, Kalyanaraman B, Finkel T, Stamler JS, Rhee SG, Vliet Avd: Redox-based regulation of signal transduction: Principles, pitfalls, and promises. Free Radic Biol Med 2008;45:1-17.

-6 Fruehauf JP, Meyskens FL: Reactive Oxygen Species: A Breath of Life or Death? Clin Cancer Res 2007;13:789.

7 Morry J, Ngamcherdtrakul W, Yantasee W: Oxidative stress in cancer and fibrosis: Opportunity for therapeutic intervention with antioxidant compounds, enzymes, and nanoparticles. Redox Biology 2016;11:240-253.

-8 Döppler H, Storz P: Mitochondrial and Oxidative Stress-Mediated Activation of Protein Kinase D1 and Its Importance in Pancreatic Cancer. Front Oncol 2017;7:41.

-9 Piskounova E, Agathocleous M, Murphy M, Hu Z, Deberardinis R, Morrison S: Abstract 2806: Oxidative stress limits metastasis of human melanoma cells. Cancer Res 2016;76:2806-2806.

10 Saha SK, Lee SB, Won J, Choi HY, Kim K, Yang GM, Dayem AA, Cho SG: Correlation between Oxidative Stress, Nutrition, and Cancer Initiation. Int J Mol Sci 2017;18:1544.

11 Hong YH, Uddin MH, Jo U, Kim B, Song J, Dong HS, Kim HS, Yong SS: ROS Accumulation by PEITC Selectively Kills Ovarian Cancer Cells via UPR-Mediated Apoptosis. Front Oncol 2015;5:167.

12 Horio Y, Hayashi T, Kuno A, Kunimoto R: Cellular and molecular effects of sirtuins in health and disease. Clin Sci 2011;121:191-203.

13 Shuang T, Wang M, Zhou Y, Shi C: Over-expression of Sirt1 contributes to chemoresistance and indicates poor prognosis in serous epithelial ovarian cancer (EOC). Med Oncol2015;32:260.

14 Teng Y, Zuo X, Hou M, Zhang Y, Li C, Luo W, Li X: A Double-Negative Feedback Interaction between MicroRNA-29b and DNMT3A/3B Contributes to Ovarian Cancer Progression. Cell Physiol Biochem 2016;39:2341.

15 Yue T, Yan Z, Kai Q, Yang X, Jing F, Wei C, Xu L: MicroRNA-29B (mir-29b) regulates the Warburg effect in ovarian cancer by targeting AKT2 and AKT3. Oncotarget 2015;6:40799-40814.

16 Teng Y, Zhao L, Zhang Y, Chen W, Li X: Id-1, a protein repressed by miR-29b, facilitates the TGFß1-induced epithelial-mesenchymal transition in human ovarian cancer cells. Cell Physiol Biochem 2014;33:717-730.

17 Oliveto S, Mancino M, Manfrini N, Biffo S: Role of microRNAs in translation regulation and cancer. World J Biol Chem 2017;8:45-56.

18 Ying X, Wei K, Lin Z, Cui Y, Ding J, Chen Y, Xu B: MicroRNA-125b Suppresses Ovarian Cancer Progression via Suppression of the Epithelial-Mesenchymal Transition Pathway by Targeting the SET Protein. Cell Physiol Biochem 2016;39:501-510.

19 Li P, Sun Y, Liu Q: MicroRNA-340 Induces Apoptosis and Inhibits Metastasis of Ovarian Cancer Cells by Inactivation of NF-kB1. Cell Physiol Biochem 2016;38:1915-1927.

20 Song N, Liu H, Ma X, Zhang S: Placental Growth Factor Promotes Metastases of Ovarian Cancer Through MiR-543-Regulated MMP7. Cell Physiol Biochem 2015;37:1104-1112.

-21 Chen Q Qin R, Fang Y, Li H: Berberine Sensitizes Human Ovarian Cancer Cells to Cisplatin Through miR-93/ PTEN/Akt Signaling Pathway. Cell Physiol Biochem 2015;36:956-965.

-22 Farooqi AA, Fayyaz S, Shatynska - Mytsyk I, Javed Z, Jabeen S, Yaylim I, Gasparri ML, Panici PB: Is miR - 34a a Well - equipped Swordsman to Conquer Temple of Molecular Oncology? Chem Biol Drug Des 2016;87:321.

23 Dai F, Zhang Y, Chen Y: Involvement of miR-29b signaling in the sensitivity to chemotherapy in patients with ovarian carcinoma. Hum Pathol 2014;45:1285-1293.

24 Xue T, Wei L, Zha DJ, Qiu JH, Chen FQ Qiao L, Qiu Y: miR-29b overexpression induces cochlear hair cell apoptosis through the regulation of SIRT1/PGC-1 $\alpha$ signaling: Implications for age-related hearing loss. Int J Mol Med 2016;38:1387-1394.

25 Xu Z, Zhang L, Fei X, Yi X, Li W, Wang Q: The miR-29b-Sirt1 axis regulates self-renewal of mouse embryonic stem cells in response to reactive oxygen species. Cell Signal 2014;26:1500-1505. doi: 10.1016/j. cellsig.2014.03.010. 


\section{Cellular Physiology Cell Physiol Biochem 2017;43:1767-1776

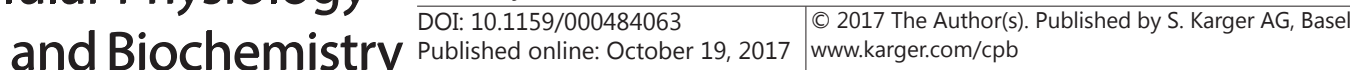

Hou et al.: Interaction of Mir-29b and SIRT1 in EOC

26 Gasparri ML, Casorelli A, Bardhi E, Besharat AR, Savone D, Ruscito I, Farooqi AA, Papadia A, Mueller MD, Ferretti E: Beyond circulating microRNA biomarkers: Urinary microRNAs in ovarian and breast cancer. Tumour Biol 2017;39:1010428317695525.

27 Zorov DB, Juhaszova M, Sollott SJ: Mitochondrial reactive oxygen species (ROS) and ROS-induced ROS release. Physiol Rev 2014;94:909.

-28 Ray PD, Huang B, Tsuji Y: Reactive oxygen species (ROS) homeostasis and redox regulation in cellular signaling. Cell Signal 2012;24:981-990.

-29 Barreiroalonso A, Lamasmaceiras M, Rodríguezbelmonte E, VizosoVázquez Á, Quindós M, Cerdán ME: High Mobility Group B Proteins, Their Partners, and Other Redox Sensors in Ovarian and Prostate Cancer. Oxid Med Cell Longev 2015;2016:5845061.

-30 Cesaratto L, Grisard E, Coan M, Zandonà L, Mattia ED, Poletto E, Cecchin E, Puglisi F, Canzonieri V, Mucignat MT: BNC2 is a putative tumor suppressor gene in high-grade serous ovarian carcinoma and impacts cell survival after oxidative stress. Cell Death Dis 2016;7:e2374.

31 Pei H, Yi Y, Lin C, Yang J, Li X, Yang Y, Duan H: Bisdemethoxycurcumin inhibits ovarian cancer via reducing oxidative stress mediated MMPs expressions. Sci Rep 2016;6:28773.

32 Qian W, Liang X, Zhang X, Bu S, Zhu X, Lai D: Autophagy protects ovarian cancer-associated fibroblasts against oxidative stress. Cell Cycle 2016;15:1376.

33 Lin J, Chuang CC, Zuo L: Potential roles of microRNAs and ROS in colorectal cancer: diagnostic biomarkers and therapeutic targets. Oncotarget 2017;8:17328.

34 Dando I, Cordani M, Dalla PE, Biondani G, Donadelli M, Palmieri M: Antioxidant Mechanisms and ROSRelated MicroRNAs in Cancer Stem Cells. Oxid Med Cell Longev 2015;2015:425708.

-35 He J, Jiang BH: Interplay between Reactive oxygen Species and MicroRNAs in Cancer. Current Pharmacology Reports 2016;2:1-9.

-36 Imai S-i, Guarente L: NAD + and sirtuins in aging and disease. Trends Cell Biol 2014;24:464-471.

-37 Imai S, Kiess W: Therapeutic potential of SIRT1 and NAMPT-mediated NAD biosynthesis in type 2 diabetes. Front Biosci 2009;14:2983.

38 Masri S: Sirtuin-Dependent Clock Control: New Advances in Metabolism, Aging and Cancer. Curr Opin Clin Nutr Metab Care 2015;18:521-527.

39 SE C, JK K: Regulation of SIRT1 by microRNAs. Mol Cells 2013;36:385.

40 Zhou L, Fu L, Lv N, Chen XS, Liu J, Li Y, Xu QY, Huang S, Zhang XD, Dou LP: A minicircuitry comprised of microRNA- 9 and SIRT1 contributes to leukemogenesis in $\mathrm{t}(8 ; 21)$ acute myeloid leukemia. Eur Rev Med Pharmacol Sci 2017;21:786.

41 Yang Y, Liu Y, Xue J, Yang Z, Shi Y, Shi Y, Lou G, Wu S, Qi J, Liu W: MicroRNA-141 Targets Sirt1 and Inhibits Autophagy to Reduce HBV Replication. Cell Physiol Biochem 2017;41:310.

42 Jr SG, Pandey S, Nedeljkovickurepa A, Saxena M, Wang A, Pruitt K: Frizzled 7 expression is positively regulated by SIRT1 and $\beta$-catenin in breast cancer cells. PLoS One 2014;9:e98861.

\$3 Pruitt K, Zinn RL, Ohm JE, Mcgarvey KM, Kang SHL, Watkins DN, Herman JG, Baylin SB: Inhibition of SIRT1 Reactivates Silenced Cancer Genes without Loss of Promoter DNA Hypermethylation. Plos Genetics 2006;2:e40.

44 Ikenoue T, Inoki K, Zhao B, Guan KL: PTEN acetylation modulates its interaction with PDZ domain. Cancer Res 2008;68:6908-6912. 